\author{
Marquette University \\ e-Publications@Marquette
}

$1-2017$

\title{
The Role Body-Esteem Plays in Impairment Associated with Hair- Pulling and Skin Picking in Adolescents
}

\author{
Elle Brennan \\ Kent State University \\ Douglas W. Woods \\ Marquette University, douglas.woods@marquette.edu \\ Martin E. Franklin \\ University of Pennsylvania \\ Nancy Keuthen \\ Massachusetts General Hospital \\ John Piacentini \\ University of California, Los Angeles
}

See next page for additional authors

Follow this and additional works at: https://epublications.marquette.edu/psych_fac

Part of the Psychology Commons

\section{Recommended Citation}

Brennan, Elle; Woods, Douglas W.; Franklin, Martin E.; Keuthen, Nancy; Piacentini, John; and Flessner, Christopher A., "The Role Body-Esteem Plays in Impairment Associated with Hair-Pulling and Skin Picking in Adolescents" (2017). Psychology Faculty Research and Publications. 286.

https://epublications.marquette.edu/psych_fac/286 


\section{Authors}

Elle Brennan, Douglas W. Woods, Martin E. Franklin, Nancy Keuthen, John Piacentini, and Christopher A. Flessner 
Marquette University

e-Publications@Marquette

\section{Psychology Faculty Research and Publications/College of Arts and Sciences}

This paper is NOT THE PUBLISHED VERSION; but the author's final, peer-reviewed manuscript. The published version may be accessed by following the link in the citation below.

Journal of Obsessive-Compulsive and Related Disorders, Vol. 12 (January, 2017): 46-51. DOI. This article is (c) Elsevier and permission has been granted for this version to appear in e-Publications@Marquette. Elsevier does not grant permission for this article to be further copied/distributed or hosted elsewhere without the express permission from Elsevier.

\section{The Role Body-Esteem Plays in Impairment Associated With Hair-Pulling and Skin Picking in Adolescents}

\section{Elle Brennan}

Department of Psychological Sciences, Kent State University, Kent, OH Douglas W. Woods

Department of Psychology, Marquette University, Milwaukee, WI

Martin E. Franklin

Department of Psychiatry, University of Pennsylvania, Philadelphia, PA

Nancy Keuthen

Trichotillomania Clinic and Research Unit, Massachusetts General Hospital, Boston, MA John Piacentini

Department of Psychiatry and Biobehavioral Sciences, University of California Los Angeles, Los Angeles, CA

Christopher A. Flessner

Department of Psychological Sciences, Kent State University, Kent, OH 
Highlights

- Body-esteem does not directly predict psychosocial functioning in youth with HPD.

- Skin picking in addition to HPD worsens adolescents' appearance-based body-esteem.

- Compounding impact of multiple BFRBs must be considered in case conceptualization.

\section{Abstract}

Trichotillomania (hair pulling disorder, HPD) and pathological skin picking (PSP) are associated with significant rates of psychosocial impairment and distress. Little research has addressed the physical consequences and associated impairment in youth (e.g., poor bodyesteem). The present study explores the relationship between body-esteem, skin picking (SP), and pulling-related impairment in a sample of adolescents with primary HPD. Ninety four adolescents who pull their hair, 40 of whom also pick their skin, were recruited via internetsampling as part of the Child and Adolescent Trichotillomania Impact Study (CA-TIP). All youth and a parent completed anonymous questionnaires online assessing psychiatric symptoms, repetitive behaviors, and psychosocial impairment, among other variables. Appearance-based body-esteem was not found to be predictive of more severe psychosocial impairment in these youth. However, SP, in combination with HPD, contributed to worse appearance-based bodyesteem above and beyond symptoms of HPD alone. The current study suggests that psychosocial functioning in youth with HPD is less impacted by body-esteem or pulling than other factors (e.g., depression and anxiety), and that SP contributes to lowered body-esteem. These findings suggest the importance of addressing body-esteem in case conceptualization for youth with both HPD and SP. Further research is required to confirm these suggestions.

Keywords

Hair-pulling disorder; Skin picking; Body-esteem; Psychosocial impairment; Adolescence

\section{Introduction}

Trichotillomania (hair pulling disorder, HPD) has been conceptualized as part of a class of body-focused repetitive behaviors (BFRBs; Mansueto, Thomas, \& Brice, 2007; Stein et al., 2008) along with excoriation disorder (also known as pathological skin picking; PSP). HPD, which occurs in approximately 1-3\% of adults (Christenson, Pyle, \& Mitchell, 1991), is characterized by chronic and excessive pulling out of one's hair that results in hair loss and clinically significant distress (American Psychiatric Association, 2013). Prevalence rates in children have not been firmly established, but HPD may be even more common in youth (Tolin et al., 2008) with the typical onset during childhood (11-13 years; Christenson, 1995; Keuthen et al., 2001). PSP is characterized by recurrent picking of the skin, resulting in skin lesions and clinically significant distress, despite repeated attempts to decrease or stop the behavior. While occasional picking of the skin is normal in the general population (Bohne et al., 2002, Hayes et al., 2009) and rates of PSP in youth are again unknown, estimated rates of clinical 
symptomatology in adults are significant (1.4-5.4\%; Hayes et al., 2009; Keuthen, Koran, Aboujaoude, Large, \& Serpe, 2010). To qualify as HPD and PSP, pulling and picking symptoms, respectively, cannot be due to another psychiatric or medical condition (APA, 2013).

Youth with HPD also exhibit greater levels of comorbid internalizing symptoms (e.g., depression, anxiety) than the general public, contributing significantly to impairment in their functioning (Lewin et al., 2009). What is more, HPD and PSP frequently co-occur (Lovato et al., 2012, Snorrason et al., 2012) - suggesting the potential for compounding levels of impairment and a shared underlying pathology (e.g., Brennan \& Flessner, 2015; Odlaug \& Grant, 2008). Available evidence for both disorders suggests impairment across several domains of functioning, including medical, emotional, occupational/academic, and psychosocial (e.g., Flessner, Conelea, et al., 2008; Harrison \& Franklin, 2012; Neziroglu, Rabinowitz, Breytman, \& Jacofsky, 2008; Odlaug \& Grant, 2008), with impairment increasing as a function of clinical severity (Begotka et al., 2004, Franklin et al., 2008, Odlaug et al., 2010). For example, youth and young adults with HPD or PSP have been found to avoid common activities (e.g., social activities, engagement with close friendships) and experience interference with academic functioning (e.g., ability to study; Franklin et al., 2008; Keuthen et al., 2000).

Psychosocial functioning is influenced by one's sense of confidence, self-efficacy, and global self-esteem. Appearance-based body-esteem, which refers to self-evaluations of one's own appearance, has been linked with global self-esteem (Mendelson, Mendelson, \& White, 2001), and thus likely also influences psychosocial functioning. Though body dissatisfaction generally emerges during childhood (McCabe \& Ricciardelli, 2003), adolescence is highlighted as a "peak" developmental period for critical evaluations of one's physical attributes (e.g., Ata, Ludden, \& Lally, 2007). In adults with HPD, low self-esteem, negative self-image, feelings of unattractiveness, and body dissatisfaction are common (Penzel, 2003, Soriano et al., 1996). Furthermore, high levels of self-criticism regarding one's appearance are related to frequency of pulling, anxiety, and depression in females with HPD (Soriano et al., 1996). Similar relationships may exist for PSP, though no such research among youths has been reported.

Such psychosocial difficulties may be exacerbated by feelings of isolation, shame, and embarrassment (Tolin et al., 2008). Accordingly, recent research has suggested that bodyesteem associated with symptoms of HPD may play a role in psychosocial impairment in adolescents (Altenburger, Tung, \& Keuthen, 2014). Altenburger and colleagues (2014) found that, in comparison to same age peers, HPD contributed to lower appearance-based bodyesteem independently of comorbid anxiety and/or depression diagnoses. The authors also noted that increased HPD severity correlated with worse appearance-based body-esteem supporting a hypothesis that the deleterious physical aspects of HPD impact body-esteem in youth. No such investigations have addressed the potential contribution of PSP, however. Moreover, some evidence suggests that adults with PSP may experience even greater psychosocial dysfunction than those with HPD due to the consequences of picking (Odlaug et al., 2010). Likewise, adults with PSP who pick on their face have been found to report greater dissatisfaction with their appearance than responders who do not pick on their face (Snorrason et al., 2013). Unfortunately, no comparisons of HPD and PSP currently exist in the youth 
literature and little is known about the impact these disorders have in combination in affected youth. The present study thus seeks to address this latter gap by examining the relationship between body-esteem and pulling-related psychosocial impairment in adolescents with HPD, or both HPD and skin picking (SP) symptoms.

When considering the literature and the conspicuous physical consequences of disorders such as HPD and PSP, it becomes apparent that youth who engage in behaviors symptomatic of these disorders are at risk for decreased self-esteem, psychosocial functioning, and overall quality of life. Following this literature, the primary aim of this paper is to explore the relationships between HPD severity, body-esteem, and pulling-related psychosocial impairment in a youth sample. Specifically, we hypothesized that appearance-based bodyesteem in youths will predict greater psychosocial impairment above and beyond the influence of HPD symptom severity and self-reported depressive and anxiety symptoms. Furthermore, little is yet understood regarding the impact on body-esteem in youth with multiple repetitive pathological behaviors (i.e., hair pulling and skin picking). As such, a secondary aim is to quantify the additional impact of co-occurring SP on appearance-based body-esteem in youth who pull their hair. To this, we hypothesized that, while controlling for additional self-reported psychiatric symptomatology, the additive combination of SP with symptoms of hair pulling (HPD+SP) will be associated with poorer appearance-based body-esteem than symptoms of hair pulling alone (HPD only).

\section{Materials and methods}

\subsection{Participants}

For the present study, a subset of adolescent participants was selected from the 336 youth recruited as part of the larger Child and Adolescent Trichotillomania Impact Project (CA-TIP; e.g., Flessner, Woods, et al., 2008; Franklin et al., 2008). The CA-TIP investigated the measureable impact of HPD symptoms on youth ages 10-17 years. Details on the distribution of the CA-TIP survey can be found in Section 2.8. Briefly, 869 individuals opened the survey, and 336 completed the majority of the survey. Cases were included in the current study if (1) both a parent and child completed all measures of interest (e.g., BESAA, described below), (2) the child was $13-17$ years of age $(M=15.27 \pm 1.38)$ and (3) the child's parents reported that their child met modified HPD diagnostic criteria (below), resulting in a final sample of 94 adolescents. For the purposes of this study (and based upon prior HPD research among youths utilizing internet-sampling procedures (e.g., Flessner et al., 2007; Franklin et al., 2008)), modified diagnostic criteria included: the child currently pulls his/her hair resulting in noticeable loss (e.g., bald patches; parent and/or child report) not due to the direction of internal voices (e.g., an imaginary friend; child report), the belief that bugs are crawling on the skin (child report), the use of illicit substances (parent and/or child report), or as the result of physical causes (e.g., skin conditions; parent report). Parental-report was the sole determinant of whether adolescents were additionally categorized as currently engaging in broadly-defined SP ("Does your child currently engage in [...] recurrent picking at the skin or scabs resulting in physical damage..."). Of note, though SP is a focal behavior for this paper, it was not an inclusion criterion during the original recruitment of participants for the CA-TIP. Ninety-four youth met modified criteria for HPD, 40 of whom also picked their skin. The sample was 
predominantly female $\left(n=85, n_{\text {male }}=9\right)$ and Caucasian $(n=79)$, with $2.1 \%(n=2)$ identifying as African American and 4.3\% (n=5) as Multi-racial. Additionally, 5.3\% $(n=5)$ identified as Hispanic/Latino, and 4.3\% $(n=4)$ declined to report their ethnicity. Additional detailed demographic characteristics can be seen in Table 1.

Table 1. Sample characteristics and descriptive statistics for primary outcome measures [Mean (Standard Deviation), unless otherwise noted].

\begin{tabular}{|clll|}
\hline \multicolumn{5}{|c}{ HPD only $(\boldsymbol{n}=\mathbf{5 4})$ HPD and SP $(\boldsymbol{n}=\mathbf{4 0})$ Whole Sample $(\boldsymbol{N}=\mathbf{9 4})$} \\
Demographics & & & \\
Sex (female, $\boldsymbol{n}(\%))$ & $50(92.6 \%)$ & $35(87.5 \%)$ & $85(90.4 \%)$ \\
Age (years) & $15.22( \pm 1.39)$ & $15.35( \pm 1.37)$ & $15.27( \pm 1.38)$ \\
Race (Caucasian, $\boldsymbol{n}(\%))$ & $46(85.2 \%)$ & $33(82.5 \%)$ & $79(84.0 \%)$ \\
Education (years) & $9.7( \pm 1.66)$ & $9.22( \pm 2.60)$ & $9.50( \pm 2.11)^{\mathrm{a}}$ \\
Family Income (median) & $\$ 50,000-75,000$ & $\$ 50,000-75,000$ & $\$ 50,000-75,000$ \\
Body-Esteem Scale for Adults and Adolescents \\
Appearance subscale & $1.91( \pm 0.97)$ & $1.48( \pm 1.00)$ & $1.73( \pm 1.00)$ \\
\multicolumn{4}{c}{ Psychosocial Impairment } \\
Social Activities & $3.91( \pm 2.51)$ & $5.30( \pm 2.56)$ & $4.50( \pm 2.64)$ \\
Social Relationships & $3.61( \pm 2.56)$ & $4.30( \pm 2.83)$ & $3.90( \pm 2.68)$ \\
Academic Functioning & $3.20( \pm 2.17)$ & $3.60( \pm 2.92)$ & $3.37( \pm 2.51)$ \\
Composite & $3.80( \pm 2.31)$ & $3.85( \pm 2.11)$ & $3.82( \pm 2.22)$ \\
Self-Reported Symptomology & & $6.79( \pm 2.59)$ \\
HPD Severity (TSC-C) & $6.83( \pm 2.33)$ & $6.73( \pm 2.94)$ \\
Depression (CDI) & $58.72( \pm 15.39)$ & $61.00( \pm 15.62)$ & $59.69( \pm 15.45)$ \\
Anxiety (MASC-C) & $54.76( \pm 13.56)$ & $56.95( \pm 15.09)$ & $55.69( \pm 14.20)$ \\
\hline
\end{tabular}

aNote: 2 Children were not in school at the time of survey completion.

\subsection{Measures}

Notably, good reliability has been published for child self-report of HPD symptoms (e.g., Franklin et al., 2008; Tolin et al., 2008) and children have more broadly been recognized as adequate reporters of internalizing symptoms (Welner et al., 1987, Yule, 1993). This is particularly true of adolescents with disorders such as HPD (Keuthen et al., 2008). As such, though group inclusion was based on parent and/or child report of pulling and only parentreport of picking, this study utilized child report of HPD symptom severity and functional impairment, as well as body-esteem, in order to maintain greater consistency in measurement. 


\subsubsection{Trichotillomania Impact Survey - Child (TIS-C)}

TIS-C (Franklin et al., 2008) utilizes parent and/or child self-report to assess demographic characteristics, HPD symptoms, related behaviors (i.e., nail biting, SP, etc.), family psychiatric history, phenomenology- and treatment-related data relevant to a child's symptoms of HPD, the impact of pulling on psychosocial impairment, and several self-/parent-report measures (incorporated within the TIS-C) designed to assess pulling severity (i.e., "On most days in the last week, how many hairs did you pull out? "), body-esteem ("My looks upset me."), and other psychiatric symptoms ("I have trouble sleeping many nights.") in greater detail. What follows is a brief description of individual items and/or measures utilized from the TIS-C for the current investigation.

Psychosocial impairment items were derived from the adolescent portion of the TIS-C. Three items were modeled after constructs assessed by the Sheehan Disability Scale (SDS; Sheehan, 2000) to ascertain aspects of psychosocial quality of life relevant to adolescents: "How much do you think your hair pulling gets in the way of doing fun things like hanging out with friends, going shopping, or going out places? "How much do you think your hair pulling gets in the way of making new friends or becoming closer to friends that you already have (like making 'best friends')? " and "How much do you think hair pulling interferes with school or your homework? " These items were rated on a 9-point Likert scale with higher scores indicative of greater impairment. A psychosocial impairment composite was calculated using the average rating across the three individual items.

\subsubsection{Trichotillomania Scale for Children - Child version (TSC-C)}

TSC-C (Tolin et al., 2008) is a 12-item measure of HPD consisting of "severity" (5 items) and "distress/impairment" (7 items) subscales. Responses are scored between 0 and 2 points and then averaged within each subscale, with higher scores indicating greater pulling severity or worse impairment. Each subscale has shown good internal consistency and validity (Cronbach's alphas 0.76 and 0.84; Tolin et al., 2008). The severity scale in particular was used in the present analyses. Internal consistency was also high in the present sample, with 0.90 for the entire TSC-C measure and 0.87 for the severity scale specifically.

\subsubsection{Body-Esteem Scale for Adolescents and Adults (BESAA)}

BESAA (Mendelson et al., 2001) is a 23-item measure with averaged subscales of general feelings regarding one's own appearance (Appearance; 10 items, e.g., "I like what I see when I look in the mirror"), one's perceptions of others' evaluations about one's body and appearance (Attribution; 5 items, e.g., "People my own age like my looks"), and weight satisfaction (Weight; 8 items, e.g., "I am satisfied with my weight"). Responses regarding agreement with item statements are provided on a 5-point Likert scale ranging from never (0) to always (4) with higher scores indicating more positive body-esteem. The three subscales have shown high internal consistency (Cronbach's alphas 0.92, 0.94, and 0.81) and good convergent reliability ( $r$ $=0.47-0.63$ ) with the Rosenberg Self-Esteem Scale (Mendelson et al., 1997). Norms for each scale have been published (Mendelson, et al., 2001), suggesting that the present sample scores below average for body-esteem (e.g., feel worse about appearance). For the current 
study, only the Appearance subscale was investigated, as it is was deemed more inclusive of a broader array of body-esteem factors which may be relevant to the sample recruited herein (i.e., youths with symptoms characteristic of HPD and SP). Further, the Weight subscale has been found to correlate highly with the Appearance subscale $(r=0.69)$ and both previous literature (e.g., Altenburger et al., 2014) and preliminary analyses have suggested that the Attributions subscale is not pertinent to youth with HPD.

\subsubsection{Children's Depression Inventory (CDI)}

CDI (Kovacs \& Beck, 1977) is a 27-item self-report measure designed to assess depressive symptoms in youth, specifically individuals ranging in age from 7 to 17 years. Item severity ranges from not a problem (0) to severe (2), with scores from all items summed into a total depression score ranging from 0 to 54 (Bang, Park, \& Kim, 2015). For the present study, raw sum scores were transformed to T-scores for ease of comparison. Due to the content of item 9 (i.e., suicidal behavior) and the prohibition of direct participant contact in the collection of data, this item was excluded from the internet survey and a pro-rated score was used in its place. This score was based on the average score obtained from the subscale associated with item 9 (Negative Self-Esteem). The CDI has been found to measure a unitary concept (Helsel \& Matson, 1984) with good psychometric properties (Smucker, Craighead, Craighead, \& Green, 1986). In the present sample, internal consistency (Cronbach's alpha) was calculated to be 0.92 for the CDI.

\subsubsection{Multidimensional Anxiety Scale for Children - Child Version (MASC-C)}

MASC-C (March, 1997) is a 39-item child self-report rating scale designed to assess a broad range of anxiety problems in children. The MASC-C consists of a total anxiety scale, an Anxiety Disorders Index, Consistency Index, and several subscales. Items are rated from never to often on a four-point scale 0-3) with higher scores indicating worse anxiety. For the present study, only T-scores for the total anxiety scale were utilized. Adequate internal consistency, test-retest reliability, and concurrent validity have been demonstrated for this measure (March, Parker, Sullivan, Stallings, \& Conners, 1997). MASC-C internal consistency was demonstrated to be high in the present sample at 0.93 .

\subsection{Procedures}

The CA-TIP was approved by the University of Wisconsin-Milwaukee Institutional Review Board (IRB). Subsequent approval for the current study was obtained from the Kent State University IRB. A surveymonkey.com link to the CA-TIP survey (TIS-C) was provided on the TLC website (www.trich.org) from May through July 2006 and was distributed via the TLC newsletter list serve. Respondents were informed prior to starting the survey that submission of the survey constituted consent to participate in research, and parents were asked to complete their section before allowing their child to complete their portion privately. 


\subsection{Data analytic plan}

Preliminary analyses revealed no significant group differences on selected demographic variables (age, sex, race, grade level, family income; $p s>0.16$ ) between youth meeting modified criteria for HPD alone $(n=54)$ and those with both symptoms characteristic of HPD and SP $(n=40)$. The HPD and HPD+SP groups were also compared on continuous measures of HPD symptom severity $(p=0.84)$ as well as other self-reported symptomatology (depression, anxiety; $p s>0.46$ ) - revealing no statistically significant differences. Assumptions germane to all following analyses were met such that no analytic modifications were necessary.

In approaching our primary aim, a composite score of the three TIP-C impairment items noted previously (see Section 2.2) was computed to assess pulling-related psychosocial impairment. Next, a hierarchical multiple regression was conducted with the Appearance subscale included as the independent variable, and the composite psychosocial impairment score as the dependent variable. Previous literature (e.g., Altenburger et al., 2014) and preliminary correlational analyses suggested HPD severity $(p<0.05)$, CDI T-score $(p<0.01)$, and MASC Tscore $(p<0.01)$ to be appropriate covariates (Step 1) for the current analyses involving the psychosocial impairment composite. The BESAA Appearance subscale was then entered in Step 2 of the model. These analyses were completed using the entire sample of youth with symptoms characteristic of HPD $(n=94)$.

To address our secondary aim, an additional hierarchical multiple regression was conducted to determine whether diagnostic status (HPD+SP [coded 1] vs. HPD only [coded 0]) predicted poorer body-esteem. Adolescents were included in the HPD+SP group if it was indicated by their reporting parent that, in addition to meeting the present study's modified diagnostic criteria for HPD, the child also reported picking his/her skin resulting in physical damage (yes/no). Adolescent age $(p<0.01)$, CDI T-score $(p<0.01)$, and/or MASC T-score $(p<0.01)$ were included as covariates (Step 1) for the current analyses involving BESAA-Appearance subscale with diagnostic status subsequently entered into Step 2 of the regression model.

A priori power analyses were conducted with regards to both aims using $G^{*}$ Power (Erdfelder, Faul, \& Buchner, 1996) based on findings from previous research conducted on the relationship between HPD and body-esteem (e.g., Altenburger et al., 2014; $d=-1.15, r$ $=-0.50$ ). Analyses suggested a sample size of approximately 89 participants was necessary to achieve power equal to 0.82 , assuming a moderate effect size. Therefore, this study's sample of 94 respondents is sufficient to adequately power both aims for this study.

\section{Results}

Descriptive statistics for the primary outcome measures (BESAA, Psychosocial Impairment), HPD symptom severity, and additional psychiatric symptomatology (depression, anxiety) for the entire sample and subgroups (HPD only, HPD+SP) are presented in table 1. 


\subsection{Aim one: Impact of body-esteem on pulling-related psychosocial impairment in youth}

with symptoms of HPD

An initial hierarchical regression revealed that the inclusion of HPD symptom severity $(\beta=0.09$, $p=0.34)$, depressive symptoms $(\beta=0.45, p<0.001)$, and anxiety symptoms $(\beta=0.19, p=0.05)$ accounted for $32.2 \%$ of the variance in psychosocial impairment, $R^{2}=0.32, F(3,90)=14.23$, $p<0.001$. The addition of appearance-based body-esteem $(\beta=-0.05, p=0.67)$ in Step 2 did not significantly contribute to the variance explained by the model, accounting for only an additional $0.1 \%$ of the variance in psychosocial impairment, $\Delta R^{2}<0.001, F(1,89)=10.62$, $p=0.67$. Additional model details are presented in $\underline{\text { Table } 2}$.

Table 2. Summary of hierarchical regression analyses for psychosocial impairment score.

\begin{tabular}{|c|c|c|c|c|c|c|}
\hline $\begin{array}{l}\text { Psychosocial Impairment } \\
\text { Analysis 1a (Appearance) }\end{array}$ & $B$ & $S E(B)$ & $\beta$ & $R^{2}$ & $\boldsymbol{F}$ & $\Delta R^{2}$ \\
\hline Step 1 & & & & 0.32 & $14.23^{* \star \star \star}$ & 0.32 \\
\hline TSC-C Severity & 0.07 & 0.08 & 0.09 & & & \\
\hline CDI Total T-Score & 0.06 & 0.01 & $0.45^{\star \star \star \star ~}$ & & & \\
\hline MASC Total T-Score & 0.03 & 0.02 & $0.19 *$ & & & \\
\hline Step 2 & & & & 0.32 & 10.62 & 0.00 \\
\hline TSC-C Severity & 0.06 & 0.08 & 0.08 & & & \\
\hline CDI Total T-Score & 0.06 & 0.02 & $0.42^{* \star * *}$ & & & \\
\hline MASC Total T-Score & 0.03 & 0.02 & 0.18 & & & \\
\hline BESAA Appearance & -0.01 & 0.03 & -0.05 & & & \\
\hline
\end{tabular}

${ }^{\star * \star} p<0.001$ (two-tailed).

${ }^{*} p<0.05$ (two-tailed).

\subsection{Aim two: Comparison of body-esteem in youth with HPD only versus HPD and SP}

A second hierarchical regression was conducted with the Appearance subscale of the BESAA as the outcome measure of interest. Per this analysis, child age $(\beta=-0.21, p=0.01)$, depressive symptoms $(\beta=-0.52, p<0.001)$, and anxiety symptoms $(\beta=-0.19, p=0.03)$ were entered into Step 1 and accounted for $47.1 \%$ of the variance in scores on the Appearance subscale of the BESAA, $R^{2}=0.47, F(3,90)=26.75, p<0.001$. The addition of diagnostic status as a predictor in Step $2(\beta=-0.15, p=0.05)$ significantly improved model fit, $\Delta R^{2}=0.30, F(1,89)=21.80, p=0.05$, explaining an additional $2.4 \%$ of the variance in BESAA-Appearance scores. This suggests that, after controlling for the influence of relevant control variables (i.e., child age, depressive symptoms, and anxiety symptoms), the additional presence of SP in adolescents exhibiting symptoms characteristic of HPD predicts lower appearance-related body-esteem compared to HPD alone. See Table 3 for additional model details. 
Table 3. Summary of hierarchical regression analyses for BESAA subscales (HPD+SP vs. HPD only).

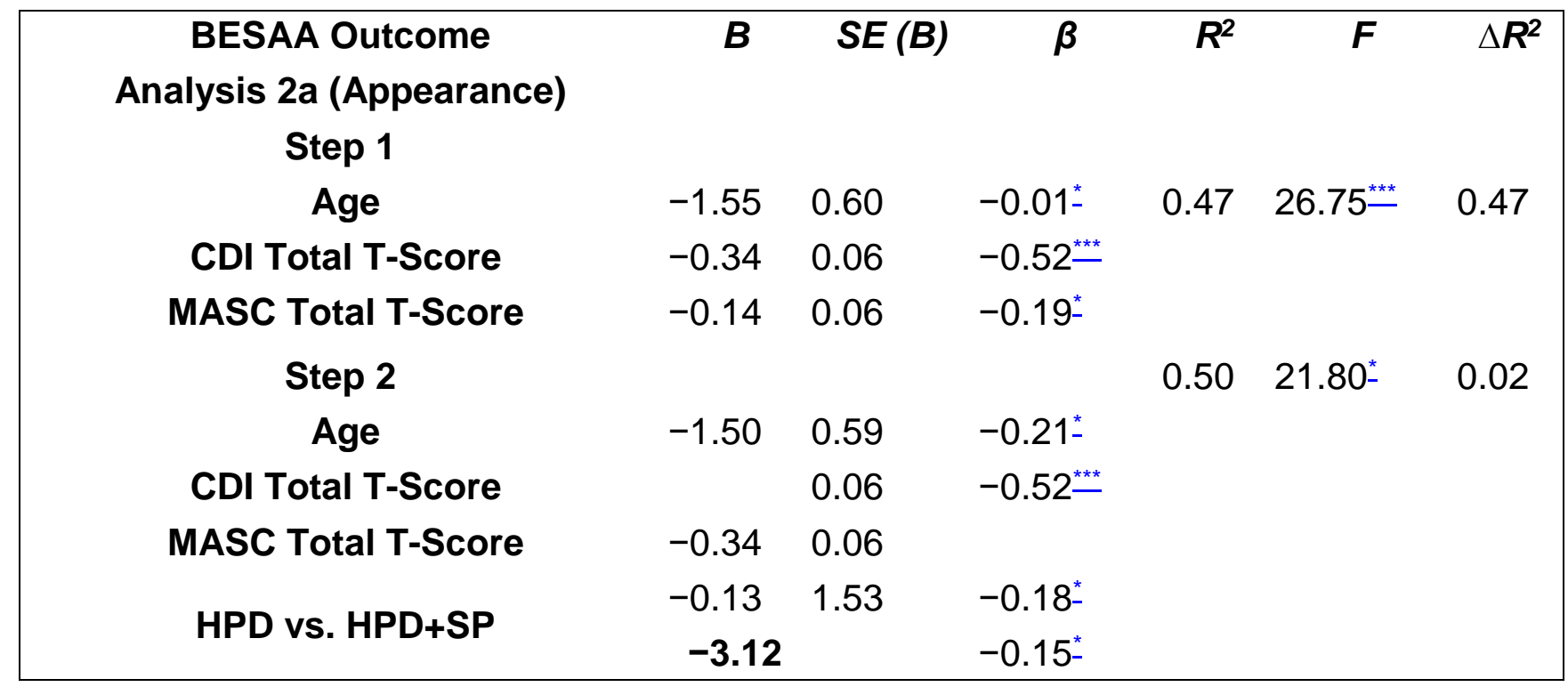

Note. Group status was coded with HPD only $=0$ and HPD+SP=1.

${ }^{* * *} p<0.001$ (two-tailed).

${ }^{*} p<0.05$ (two-tailed).

\section{Discussion}

The current study sought to better characterize the relationships between appearance-related body-esteem, psychosocial impairment, and symptoms characteristic of HPD and SP in adolescents. Two approaches were taken to achieve this goal, and were met with mixed results. The first involved testing whether pulling-related psychosocial impairment is driven by body-esteem above and beyond psychiatric symptomatology (i.e., HPD severity, depression, anxiety). The second focused on understanding whether or not symptoms of SP, over and above the presence of symptoms of HPD, significantly predict body-esteem ratings in adolescents with symptoms characteristic of HPD. What follows is a brief review and discussion of these findings and their implications.

Pulling-related psychosocial impairment in this sample of adolescents with HPD was less related to appearance-based body-esteem - and symptom severity - than to depressive symptomatology and anxiety (see Table 2). Adolescents with symptoms characteristic of HPD are typically mindful of the marring of their appearance due to pulling (theoretically reflected in the Appearance subscale). However, it is possible that the impact of poor body-esteem is buffered by the secretive nature of HPD (e.g., Tolin et al., 2008), and successful avoidance of discovery (e.g., masking bald patches). Unfortunately, using avoidance strategies to prevent discovery may increase anxiety and depression, which in turn may lead to psychosocial impairment. It is important for clinicians to address comorbid anxiety and depression in cases of HPD for this and other reasons. Further, it is possible that age-related (i.e., pubertal) changes in appearance associated with this age range (e.g., weight, acne, body hair) 
contributed to ratings of body-esteem above pulling, thus skewing the impact of body-esteem on pulling-related impairment. Though these suggestions are purely speculative, it serves to emphasize the need for more in-depth investigations to better delineate the state of attributions of body-esteem in youth with HPD and similar disordered symptomology, as well as the normative challenges to body-esteem experienced during this developmental period.

Similar to prior research in adults with HPD or PSP (Odlaug et al., 2010), the deleterious symptoms of SP, in combination with HPD, contributed to worse (i.e., lower) scores on the BESAA Appearance subscale. Though these findings are limited in robustness, they suggest that the combination of scabbing and scarring due to SP and the physical consequences of HPD heightens youth's negative self-evaluations of their appearance above and beyond the impact of HPD alone. One additional factor to consider is the possibility that youth judged to engage in repetitive, damaging SP may have also been more prone to acne than those who did not engage in SP, thus impacting their scores on the BESAA. Though no causal conclusions may be drawn from the present study, several disorder-specific factors may influence these findings, such as increased difficulty of hiding the scabs and scars on open skin (e.g., face, arms) characteristic of a youth with SP, increased distress from hiding evidence of both pulling and picking, and/or frustration or disappointment at lacking self-control (e.g., engaging in a second repetitive behavior).

Given the findings of the current study, it may be prudent to consider these findings when approaching treatment of HPD and PSP in youth. Though the focused integration of building body-esteem - or more broadly self-esteem - into psychological treatment has not been empirically studied in BFRBs as of yet, a recent case study has suggested the benefits of selfesteem boosting cosmetic intervention in nail biting (Juneja, Juneja, \& Sultan, 2016).

Psychoeducational and direct therapeutic (e.g., role playing, problem-solving training) efforts to improve self-esteem in youth with a variety of difficulties (e.g., behavioral, personality) have also been reported to be beneficial (Haney and Durlak, 1998, O'Dea and Abraham, 2000). It is thus also possible that self-esteem-enhanced treatment approaches in youth with PSP and HPD (e.g., integration of positive self-talk, encouragement of self-efficacy, and improved perspective taking along with Habit Reversal Training, HRT) might alleviate avoidance

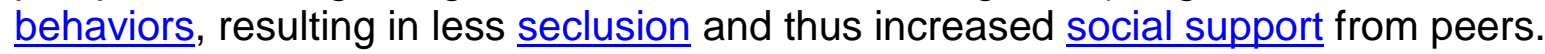

Though the current investigation suggests the importance of considering the impact of SP symptoms on the well-being of adolescents with symptoms characteristic of HPD, several limitations should be considered. First, the anonymous internet sample utilized for this study may not best represent youth with clinically significant HPD and/or PSP. However, evidence suggests that the utilization of internet samples - at least in adults - produces results that are consistent with face-to-face data collection (Wetterneck, Woods, Norberg, \& Begotka, 2006).

The lack of a control condition also limits our conclusions as we are unable to draw conclusions about the HPD group relative to "normative" peers. Further, with regards to the cross-sectional nature of the current study, future longitudinal data collection could also allow for the exploration of causal relationships in body-esteem, pulling-related psychosocial impairment, and symptom severity in youth with HPD and/or SP symptomatology (e.g. mediational effects). Research into the potential cyclical nature of these relationships would also be beneficial in determining whether there are key aspects that drive specific symptoms 
(e.g., HPD and SP impacting BE, BE increases negative affect, negative affect impacts pulling/picking frequency). Methodology such as ecological momentary assessment may be useful in tracking such patterns. Lastly, though the present sample characteristics are comparable to the extant literature (e.g., Altenburger et al., 2014), much of what research exists is generally restricted in its demographic diversity (e.g., race, sex). While aspects of this homogeneity are to be expected (i.e., HPD predominantly occurs in females; $\underline{\text { Christenson \& }}$ Crow, 1995), the potential afforded by anonymous sampling in addition to the TLC listserv (e.g., random survey mailing, utilization of Amazon Mturk) to recruit vast numbers of participants could be utilized in future research on body-esteem in youth with HPD to better identify unique patterns that might emerge in larger, more diverse samples. An additional future line of research includes recruiting a sample of youth with PSP only in order to better compare the impact of HPD to that of PSP in affected youth.

Despite these limitations, the results of the current study provide unique and interesting information regarding the impact of symptoms characteristic of HPD and comorbid SP in adolescents - through body-esteem in particular but also more broadly across pulling-related psychosocial impairment. The importance of body-esteem and the additive impact of multiple repetitive behaviors should be considered in future case conceptualization and treatment planning. Additionally, future clinical work in youth with these combined problems might aim to incorporate improvements in body-esteem as a goal of therapy to help combat shame and embarrassment and reduce avoidance, ultimately to decrease distress and improve overall impairment.

\section{References}

$1 \mathrm{E}$. Altenburger, E. Tung, N. KeuthenBody-esteem in adolescent hair pullers Journal of Behavioral Addictions, 3 (2) (2014), pp. 124-127

2 American Psychiatric Association (2013). Diagnostic and statistical manual of mental disorders (5th ed.). Washington, DC.

3 R.N. Ata, A.B. Ludden, M.M. LallyThe effects of gender and family, friend, and media influences on eating behaviors and body image during adolescence Journal of Youth and Adolescence, 36 (8) (2007), pp. 1024-1037

4 Y.R. Bang, J.H. Park, S.H. KimCut-off scores of the children's depression inventory for screening and rating severity in Korean adolescents Psychiatry Investigation, 12 (1) (2015), pp. 23-28

5 A.M. Begotka, D.W. Woods, C.T. WetterneckThe relationship between experiential avoidance and the severity of trichotillomania in a nonreferred sample Journal of Behavior Therapy and Experimental Psychiatry, 35 (1) (2004), pp. 17-24

6 A. Bohne, S. Wilhelm, N.J. Keuthen, L. Baer, M.A. JenikeSkin picking in German students prevalence, phenomenology, and associated characteristics Behavior Modification, 26 (3) (2002), pp. 320-339

$7 \mathrm{E}$. Brennan, C. FlessnerAn interrogation of cognitive findings in pediatric obsessivecompulsive and related disorders Psychiatry Research, 227 (2) (2015), pp. 135-143

8 G. ChristensonTrichotillomania - From prevalence to comorbidity Psychiatric Times, 12 (9) (1995), pp. 44-48 
9 G.A. Christenson, S.J. CrowThe characterization and treatment of trichotillomania The Journal of Clinical Psychiatry, 57 (1995), pp. 42-47

10 G.A. Christenson, R.L. Pyle, J.E. MitchellEstimated lifetime prevalence of trichotillomania in college students Journal of Clinical Psychiatry, 52 (10) (1991), pp. 415-417

11 E. Erdfelder, F. Faul, A. BuchnerGPOWER: A general power analysis program Behavior Research Methods, Instruments, \& Computers, 28 (1) (1996), pp. 1-11

12 C.A. Flessner, C.A. Conelea, D.W. Woods, M.E. Franklin, N.J. Keuthen, S.E. CashinStyles of pulling in trichotillomania: Exploring differences in symptom severity, phenomenology, and functional impact Behaviour Research and Therapy, 46 (3) (2008), pp. 345-357, 10.1016/j.brat.2007.12.009

13C.A. Flessner, D.W. Woods, M.E. Franklin, N.J. Keuthen, J. Piacentini, TLC-SABStyles of pulling in youths with trichotillomania: Exploring differences in symptom severity, phenomenology, and comorbid psychiatric symptoms Behaviour Research and Therapy, 46 (9) (2008), pp. 1055-1061, 10.1016/j.brat.2008.06.006

14 C.A. Flessner, D.W. Woods, M.E. Franklin, N.J. Keuthen, J. Piacentini, S.E. Cashin, P.S. MooreThe Milwaukee Inventory for Styles of Trichotillomania-Child Version (MISTC): Initial development and psychometric properties Behavior Modification, 31 (6) (2007), pp. 896-918, 10.1177/0145445507302521

15 M.E. Franklin, C.A. Flessner, D.W. Woods, N.J. Keuthen, J.C. Piacentini, P. Moore, TLCSABThe Child and Adolescent Trichotillomania Impact Project: Descriptive psychopathology, comorbidity, functional impairment, and treatment utilization Journal of Developmental and Behavioral Pediatrics, 29 (6) (2008), pp. 493-500

16 P. Haney, J.A. DurlakChanging self-esteem in children and adolescents: A metaanalytical review Journal of Clinical Child Psychology, 27 (4) (1998), pp. 423-433

17 J.P. Harrison, M.E. FranklinPediatric trichotillomania Current Psychiatry Reports, 14 (3) (2012), pp. 188-196, 10.1007/s11920-012-0269-8

18 S.L. Hayes, E.A. Storch, L. BerlangaSkin picking behaviors: An examination of the prevalence and severity in a community sample Journal of Anxiety Disorders, 23 (3) (2009), pp. 314-319, 10.1016/j.janxdis.2009.01.008

19 W.J. Helsel, J.L. MatsonThe assessment of depression in children: The internal structure of the Child Depression Inventory (CDI) Behaviour Research and Therapy, 22 (3) (1984), pp. 289-298

20 A. Juneja, A. Juneja, A. SultanNail Biting: A body focused repetitive behaviour case report Journal of Behavioral Health, 5 (1) (2016), pp. 33-37, 10.5455/jbh.20150826024242

21 N. Keuthen, C. Flessner, D. Woods, M. Franklin, J. Piacentini, M. Khanna, TLCSABParent-youth rating concordance for hair pulling variables, functional impairment, and anxiety scale scores in trichotillomania Child \& Family Behavior Therapy, 30 (4) (2008), pp. 337-353, 10.1080/07317100202423215

22 N.J. Keuthen, L.M. Koran, E. Aboujaoude, M.D. Large, R.T. SerpeThe prevalence of pathologic skin picking in US adults Comprehensive Psychiatry, 51 (2) (2010), pp. 183-186, 10.1016/j.comppsych.2009.04.003 
23 N.J. Keuthen, T. Deckersbach, S. Wilhelm, E. Hale, C. Fraim, L. Baer, M.A. JenikeRepetitive skin-picking in a student population and comparison with a sample of self-injurious skin-pickers Psychosomatics, 41 (3) (2000), pp. 210-215, 10.1176/appi.psy.41.3.210

24 N.J. Keuthen, T. Deckersbach, S. Wilhelm, I. Engelhard, A. Forker, R.L. O'sullivan, L. BaerThe Skin Picking Impact Scale (SPIS): Scale development and psychometric analyses Psychosomatics, 42 (5) (2001), pp. 397-403

25 M. Kovacs, A.T. BeckAn empirical-clinical approach toward a definition of childhood depression

26 J.G. Schulterbrandt, Askin (Eds.), Depression in childhood: Diagnosis, treatment, and conceptual models, Raven Press, New York (1977), pp. 1-25

27 A. Lewin, J. Piacentini, C. Flessner, D. Woods, M. Franklin, N. Keuthen, TLCSABDepression, anxiety, and functional impairment in children with trichotillomania Depression and Anxiety, 26 (2009), pp. 521-527

28 L. Lovato, Y.A. Ferrao, D.J. Stein, R.G. Shavitt, L.F. Fontenelle, A. Vivan, A.V. CordioliSkin picking and trichotillomania in adults with obsessive-compulsive disorder Comprehensive Psychiatry, 53 (5) (2012), pp. 562-568, 10.1016/j.comppsych.2011.06.008

29 C.S. Mansueto, A.M. Thomas, A.L. BriceHair pulling and its affective correlates in an African-American university sample Journal of Anxiety Disorders, 21 (4) (2007), pp. 590-599

$30 \mathrm{~J}$. MarchManual for the Multidimensional Anxiety Scale for Children (MASC) MultiHealth Systems, Toronto (1997)

31 J.S. March, J.D. Parker, K. Sullivan, P. Stallings, C.K. ConnersThe Multidimensional Anxiety Scale for Children (MASC): Factor structure, reliability, and validity Journal of the American Academy of Child and Adolescent Psychiatry, 36 (4) (1997), pp. 554-565

32 M.P. McCabe, L.A. RicciardelliSociocultural influences on body image and body changes among adolescent boys and girls The Journal of Social Psychology, 143 (1) (2003), pp. 5-26

33 B.K. Mendelson, M.J. Mendelson, D.R. WhiteBody-esteem scale for adolescents and adults Journal of Personality Assessment, 76 (1) (2001), pp. 90-106, 10.1207/S15327752JPA7601 6

34 F. Neziroglu, D. Rabinowitz, A. Breytman, M. JacofskySkin picking phenomenology and severity comparison Primary Care Companion to the Journal of Clinical Psychiatry, 10 (4) (2008), p. 306

35 J.A. O'Dea, S. Abrahamimproving the body image, eating attitudes, and behaviors of young male and female adolescents: A new educational approach that focuses on self-esteem International Journal of Eating Disorders, 28 (1) (2000), pp. 43-57

36 B.L. Odlaug, J.E. GrantClinical characteristics and medical complications of pathologic skin picking General Hospital Psychiatry, 30 (1) (2008), pp. 61-66, 10.1016/i.genhosppsych.2007.07.009 
37 B.L. Odlaug, S.W. Kim, J.E. GrantImpairment and clinical severity in pathological skin picking and trichotillomania Journal of Anxiety Disorders, 24 (8) (2010), pp. 823-829

$38 \mathrm{~F}$. PenzelThe hair-pulling problem: A complete guide to trichotillomania Oxford University Press, Oxford (2003)

39 D. SheehanSheehan Disability Scale Handbook of psychiatric measures (2000), pp. 113115

40 M.R. Smucker, W.E. Craighead, L.W. Craighead, B.J. GreenNormative and reliability data for the Children's Depression Inventory Journal of Abnormal Child Psychology, 14 (1) (1986), pp. 25-39

$41 \mathrm{I}$. Snorrason, E.L. Belleau, D.W. WoodsHow related are hair pulling disorder (trichotillomania) and skin picking disorder? A review of evidence for comorbidity, similarities, and shared etiology Clinical Psychology Review, 32 (7) (2012), pp. 618-629, 10.1016/j.cpr.2012.05.008

42 I. Snorrason, R.P. Olafsson, C.A. Flessner, N.J. Keuthen, M.E. Franklin, D.W. WoodsThe Skin Picking Impact Scale: Factor structure, validity and development of a short version Scandinavian Journal of Psychology, 54 (2013), pp. 344-348

43 J.L. Soriano, R.L. O'Sullivan, L. Baer, K.A. Phillips, R.J. McNally, M.A. JenikeTrichotillomania and self-esteem: A survey of $\mathbf{6 2}$ female hair pullers The Journal of Clinical Psychiatry, 57 (2) (1996), pp. 77-82

44 D.J. Stein, C.A. Flessner, M. Franklin, N.J. Keuthen, C. Lochner, D.W. Woodsls trichotillomania a stereotypic movement disorder? An analysis of body-focused repetitive behaviors in people with hair-pulling Annals of Clinical Psychiatry, 20 (4) (2008), pp. 194-198

45 D.F. Tolin, G.J. Diefenbach, C.A. Flessner, M.E. Franklin, N.J. Keuthen, P. Moore, TLCSABThe trichotillomania scale for children: Development and validation Child Psychiatry and Human Development, 39 (3) (2008), pp. 331-349, 10.1007/s10578-007$\underline{0092-3}$

46 Z. Welner, W. Reich, B. Herjanic, K.G. Jung, H. AmadoReliability, validity, and parentchild agreement studies of the Diagnostic Interview for Children and Adolescents (DICA) Journal of the American Academy of Child and Adolescent Psychiatry, 26 (5) (1987), pp. 649-653

47 C.T. Wetterneck, D.W. Woods, M.M. Norberg, A.M. BegotkaThe social and economic impact of trichotillomania: Results from two nonreferred samples Behavioral Interventions, 21 (2) (2006), pp. 97-109

$48 \mathrm{~W}$. YuleDevelopmental considerations in child assessment

49 T.H. Ollendick, M. Hersen (Eds.), Handbook of child and adolescent assessment. General psychology series, 167, Allyn \& Bacon, Needham Heights, MA (1993), pp. 15-25 